\title{
RELAÇÃO ENTRE A MODULARIDADE NO PROJETO E NA PRODUÇÃO EM UMA MONTADORA AUTOMOTIVA NO BRASIL
}

Flávio Issao Kubota (flavio.kubota@posgrad.ufsc.br) - Programa de Pós-Graduação em Engenharia de Produção (PPGEP), Universidade Federal de Santa Catarina (UFSC)

Juliana Hsuan (jh.om@cbs.dk) - Department of Operations Management, Copenhagen Business School, Dinamarca

Paulo Augusto Cauchick-Miguel (paulo.cauchick@ufsc.br) - Departamento de Engenharia de Produção e Sistemas (DEPS), UFSC

\section{RESUMO}

A modularidade no projeto e na produção são os principais tipos aplicados por empresas do setor automotivo. A relação entre esses tipos de modularidade tem gerado interesse crescente na academia e na indústria. Assim, o presente trabalho examina as possíveis relações entre modularidade de projeto e produção em uma montadora automotiva. A análise é conduzida por um modelo conceitual construído com base na literatura englobando os principais construtos norteadores destes tipos de modularidade. $O$ trabalho empírico realizou coleta de dados por meio de questionários e posteriores entrevistas com gestores e engenheiros de projeto e produção de uma montadora de veículos que aplica os dois tipos de modularidade. O trabalho identificou que: há potenciais benefícios obtidos pela montadora ao associar a modularidade de projeto com a modularidade de produção, e; há limitações nas relações entre esses tipos de modularidade, principalmente devido a um entendimento distinto entre as áreas de engenharia do produto e processo acerca da estratégia modular. Sugere-se que a modularidade aplicada no projeto e nos processos, de forma integrada, pode conduzir a benefícios nas fases de projeto, com decisões baseadas nas capacidades atuais e potenciais da empresa, e de produção, adequando as capacidades instaladas às necessidades da plataforma modular.

Palavras chave: Modularidade; modularidade de projeto; modularidade de produção; Setor automotivo.

Área: Gestão do Processo de Desenvolvimento de Produtos

\section{INTRODUÇÃO}

A modularidade é uma abordagem de projeto que proporciona suporte às empresas no uso de diferentes tecnologias e tomadas de decisões que envolvem produtos e processos produtivos (LUCARELLI et al., 2015). A modularidade tem crescido em termos de importância nas empresas de manufatura, especialmente em montadoras automotivas, que estão aplicando o conceito de forma crescente em novos projetos (SHAMSUZZOHA et al., 2010).

Nesse cenário, um tópico emergente está atraindo interesse de acadêmicos e profissionais do setor automobilístico: a relação entre a modularidade de projeto e de produção. Estudos empíricos que investiguem o relacionamento entre essas tipologias da modularidade ainda são escassos, embora alguns pesquisadores estejam apontando a relevância do estudo da relação entre modularidade de projeto e de produção (por exemplo, CAMPAGNOLO; CAMUFFO, 2010; JACOBS et al., 2011; SANCHEZ, 2013; LUCARELLI et al., 2015). 
Nesse sentido, algumas dessas publicações (e.g. CAMPAGNOLO; CAMUFFO, 2010; JACOBS et al., 2011; SANCHEZ, 2013; LUCARELLI et al., 2013) alegam que essas relações ainda não estão plenamente definidas na literatura e na prática. Argumenta-se que as trajetórias das relações entre os tipos de modularidade envolvidos (projeto e produção) dependeriam do objeto de análise considerado (FIXSON; PARK, 2008). Essa situação seria motivadora para analisar como a modularidade pode afetar o projeto simultâneo de produtos e sistemas de produção (CAMPAGNOLO; CAMUFFO, 2010), uma vez que o impacto do projeto modular em processos produtivos é considerado um campo interessante de pesquisas futuras (JACOBS et al., 2011).

Acrescenta-se também que pesquisas empíricas que investiguem as possíveis relações entre a modularidade de projeto e de produção, bem como seus impactos técnicos e organizacionais nas empresas automotivas que aplicam a estratégia modular, ainda são escassas (JACOBS et al., 2011; LUCARELLI et al., 2015). Tal cenário demanda mais pesquisas sobre a modularidade nesse segmento. Considerando esse contexto, este trabalho tem como objetivo investigar a relação entre a modularidade de projeto e de produção, visando identificar e analisar quais os principais elementos conceituais envolvidos nesse relacionamento, e como esses constructos norteiam a relação entre o projeto de produto e os processos produtivos, à luz da estratégia modular.

Após esta introdução, a seção 2 apresenta a fundamentação teórica desenvolvida para analisar a conexão entre modularidade de projeto e produção, que resultou em um modelo conceitual como base para a análise. Em seguida, a seção 3 apresenta-se os procedimentos metodológicos que viabilizaram o desenvolvimento e condução da investigação. Por fim, as seções 4, 5 e 6, respectivamente, apresentam os resultados, as discussões decorrentes dos mesmos, e as conclusões a partir do trabalho desenvolvido.

\section{FUNDAMENTAÇÃO TEÓRICA}

Este trabalho investiga, empiricamente, a relação entre a modularidade de projeto e produção. Nesse contexto, sua fundamentação teórica se sustenta pela literatura acerca das relações teóricas entre os tipos de modularidade de projeto e produção no setor automotivo. Para fins de melhorar o entendimento deste estudo, a partir desta seção a modularidade de projeto será mencionada como MID (sigla para modularity in design) enquanto a modularidade de produção será exposta como MIP (sigla para modularity in production). Essa nomenclatura é utilizada por outros autores, tais como Sanchez (2013) e Lucarelli et al. (2015).

Assim, esta seção é subdividida em (i) MID conduzindo a MIP; (ii) MIP conduzindo a MID; e (iii) Relação bidirecional entre MID-MIP.

\subsection{MID conduzindo a MIP}

A respeito da trajetória da relação da MID levando a MIP, alguns estudos (e.g. HOETKER 2006; PARALIKAS et al., 2011) argumentam que as decisões de modularidade durante as fases de projeto de produto afetam as decisões futuras relativas aos processos de fabricação, permitindo a aplicação da MIP. Nesse caso, a estrutura do produto influencia a sua produção, uma vez que as empresas precisam organizar seus processos de fabricação para fornecer, de forma ágil, todas as variantes do produto desenvolvidas (PARALIKAS et al., 2011). Além disso, os produtos modulares podem facilitar o redesenho organizacional das empresas, contribuindo com a flexibilidade organizacional (HOETKER, 2006).

Nessa trajetória de MID conduzindo a MIP, estão envolvidos os seguintes conceitos: outsourcing (terceirização), plataforma de produto, funcionalidade, variedade de produtos, comunalidade e interdependência entre módulos. Há tradeoffs a serem considerados nesses 
conceitos. Por exemplo, a terceirização pode ser benéfica para as montadoras e os fornecedores, no entanto a perda de aprendizado desenvolvendo atividades além da necessária para manter o conhecimento específico de componentes pode representar um limite para a terceirização de projetos de engenharia (ZIRPOLI; BECKER, 2011).

A funcionalidade é aplicada dentro do conceito de plataforma de produtos para definir como os módulos serão compostos de acordo com a arquitetura dos veículos e as funções dos módulos e subsistemas que compõem o veículo como um todo (RO et al., 2007). A partir das funcionalidades, é possível também orientar a definição da variedade de produtos, e por meio dessa decisão, as arquiteturas modulares possibilitam a formação de famílias de produtos, não apenas projetos de produtos individuais (STONE et al., 2000).

A comunalidade (do termo em inglês commonality) é um conceito geralmente mais relacionado a produtos do que processos, o qual explora a ideia de usar componentes idênticos em uma configuração de produto, mas em produtos diferentes (FIXSON, 2007). Em termos de variedade de produtos, a similaridade pode criar outras dificuldades: Pasche e Sköld (2012) argumentam que os produtos podem se tornar muito semelhantes, com maior grau de uniformidade entre diferentes produtos e/ou marcas.

A interdependência entre módulos é um conceito que é influenciado por outros elementos conceituais, especialmente a padronização e a funcionalidade. Se os módulos interagem apenas entre interfaces padronizadas, as interdependências devem ser estruturadas antes de iniciar o processo de design e não no final. Caso contrário, um redesenho de produtos e processos será inevitável e oneroso para a empresa (ZIRPOLI; BECKER, 2011).

\subsection{MIP conduzindo a MID}

Nesta trajetória da relação entre MID e MIP, alguns autores (por exemplo, TAKEISHI; FUJIMOTO, 2003; RO et al., 2007) afirmam que a produção modular é que deve ser considerada antes da arquitetura modular de produto. Nessa perspectiva, determinado tipo de arquitetura de produto é condicionado pelas capacidades organizacionais de cada empresa (RO et al., 2007). Da MIP para MID, os principais elementos conceituais que norteiam essa relação são o outsourcing (terceirização), co-design com fornecedores e a padronização.

Em relação à terceirização, os fornecedores são geralmente envolvidos nos processos de fabricação, bem como nas fases iniciais do processo de desenvolvimento do produto. Mudanças na hierarquia dos sistemas de produção geram tensão em suas relações com a arquitetura do produto, incentivando assim a redefinição da arquitetura do produto (TAKEISHI; FUJIMOTO, 2003). Além disso, o co-design com fornecedores é uma atividade onde os fornecedores podem ser fortemente envolvidos no processo de desenvolvimento de produto juntamente com as montadoras, especialmente se esses fornecedores forem de primeiro nível na cadeia de suprimento. Por fim, a MIP demanda uma robusta padronização para favorecer o redesenho do processo e/ou a inclusão ágil de novos módulos para atender às mudanças nos requisitos do produto (MIKKOLA; 2006).

\subsection{Relação bidirecional entre MID e MIP}

A terceira situação possível acontece quando os elementos conceituais envolvidos na relação entre MID-MIP estabelecem uma trajetória de bidirecional (ver FIXSON; PARK, 2008; TAKEISHI; FUJIMOTO, 2003). Assim, as relações entre arquiteturas de produtos e sistemas entre empresas podem não só ter a MID influenciando a MIP, mas também a MIP ter algum impacto sobre a MID (TAKEISHI; FUJIMOTO, 2003). Além disso, as trajetórias das relações entre MID e MIP dependem da unidade de análise considerada (FIXSON; PARK, 2008). 
Por meio da padronização, as especificações de projeto e as tolerâncias dos módulos são estabelecidas durante as etapas iniciais do processo de desenvolvimento do produto, minimizando a variabilidade nos processos de fabricação (MIKKOLA, 2006). Nesse sentido, a plataforma de produto é um grande conjunto de componentes de produto com interfaces padronizadas, fisicamente conectadas como um subconjunto estável que podem ser compartilhadas entre diferentes produtos finais, permitindo a redução do tempo e do custo de desenvolvimento, melhorando a qualidade e confiabilidade do produto, permitindo a customização em massa e, finalmente, aumentando a flexibilidade de fabricação (SANCHEZ, 2013).

Dessa forma, observa-se que as relações entre MID e MIP variam conforme o contexto e os objetivos da empresa. Nesse sentido, as capacidades e limitações de fabricação serão consideradas antes de se mudar o projeto de produto modular. A próxima seção apresenta os métodos de pesquisa para a viabilização da pesquisa de campo.

\section{MÉTODOS DE PESQUISA}

Os procedimentos metodológicos foram determinados considerando a natureza do objetivo deste trabalho e o referencial teórico sobre a relação entre MID e MIP no setor automotivo. Pouco se sabe sobre as implicações da arquitetura do produto no design organizacional, tanto dentro da empresa como em toda a cadeia de suprimentos, no contexto de mudanças em direção a uma arquitetura de produto mais modular (RO et al., 2007; JACOBS et al., 2011; LUCARELLI et al., 2015). Tal panorama sugere a demanda por mais pesquisas sobre modularidade nesse segmento industrial.

Para isso, definiu-se a abordagem de estudo de caso como método de investigação, pois são apropriados para pesquisas que demandam mais aprofundamento de determinado tema, bem como para trabalhos que possuam questão de pesquisa incorporando elementos explanatórios (VOSS, 2009; YIN, 2014), como é o caso do presente estudo. Para a seleção da empresa a ser investigada (denominada Empresa A por questão de confidencialidade), utilizou-se critérios de seleção objetivos, conforme recomendado por Sousa e Voss (2001). Nesse sentido, as principais razões para a seleção da montadora em estudo foram:

a) O fato de a empresa ter certa autonomia para desenvolver projetos locais;

b) Possuir engenheiros, gestores e/ou diretores que estiveram envolvidos nas fases iniciais do processo de desenvolvimento de produtos, fato importante para o entendimento da relação entre MID e MIP;

c) Ter fornecedores modulistas para desenvolver módulos e/ou componentes;

d) Possuir cerca de 10 anos de experiência na aplicação da modularidade, o que sugere certa maturidade quanto à estratégia modular, e;

e) Permitir acesso aos dados para coleta (descrita a seguir), embora com algumas restrições, como não permitir gravação em áudio.

\subsection{Procedimentos de coleta e análise dos dados}

Para a coleta de dados, buscou-se selecionar entrevistados que estiveram envolvidos de forma mais aproximada com as atividades e processos e relacionados a aplicação da modularidade (conforme recomendado por BARDIN, 1977). Assim, foram entrevistados executivos e engenheiros, envolvidos com o projeto de produto e as decisões estratégicas relacionadas às plataformas de produto da montadora. Os selecionados para serem entrevistados foram o gerente de Departamento de Plataformas e Sistemas, o diretor do Departamento de 
Engenharia de Projetos e um engenheiro de projetos, que também estava envolvido com a estruturação e adequação das linhas de manufatura aos projetos modulares. Cada entrevista durou cerca de uma hora e meia e os dados foram registrados por meio de anotações em papel, uma vez que a empresa não permitiu a utilização de gravação em áudio. Posteriormente, questões adicionais foram enviadas por e-mail e feitas por telefone aos entrevistados, para esclarecer algumas dúvidas sobre os dados e também para buscar respostas de outras questões emergentes durante a análise. Além disso, dados secundários tais como informações do website da empresa e informações sobre a nova plataforma de produtos da mesma foram coletados e posteriormente considerados na análise. A Tabela 1 apresenta o perfil dos entrevistados na montadora investigada, bem como uma síntese das informações relevantes que puderam oferecer.

Tabela 1. Relação dos entrevistados na Empresa A

\begin{tabular}{c|c|c}
\hline Cargo do Entrevistado & Função & $\begin{array}{c}\text { Relevância do entrevistado para a } \\
\text { pesquisa }\end{array}$ \\
\hline $\begin{array}{c}\text { Diretor do Departamento de } \\
\text { Engenharia e Projetos }\end{array}$ & $\begin{array}{c}\text { Gestão e tomada de decisão } \\
\text { sobre projetos de engenharia da } \\
\text { organização }\end{array}$ & $\begin{array}{c}\text { Informações relacionadas à gestão } \\
\text { organizacional e estratégica da } \\
\text { modularidade de projeto e produção na } \\
\text { empresa }\end{array}$ \\
\hline $\begin{array}{c}\text { Gerente do Departamento de } \\
\text { Plataformas e Sistemas }\end{array}$ & $\begin{array}{c}\text { Gestão estratégica e técnica da } \\
\text { engenharia da plataforma do } \\
\text { produto e sistemas }\end{array}$ & $\begin{array}{c}\text { Entendimento das definições técnicas de } \\
\text { engenharia e das relações entre MID e } \\
\text { MIP, a partir da plataforma de produtos da } \\
\text { empresa }\end{array}$ \\
\hline Engenheiro de Projetos & $\begin{array}{c}\text { Desenvolver projetos de } \\
\text { engenharia e análise de } \\
\text { adequação do mesmo à } \\
\text { produção }\end{array}$ & $\begin{array}{c}\text { Informações relacionadas ao } \\
\text { comportamento da plataforma de produto } \\
\text { na produção (adequação dos projetos às } \\
\text { demandas produtivas) }\end{array}$ \\
\hline
\end{tabular}

Os principais elementos conceituais envolvidos nas relações MID e MIP foram extraídos a partir das entrevistas seguindo, de forma combinada, as seguintes diretrizes de Bardin (1977), Miles e Huberman (2014), e Yin (2014) para análise de conteúdo:

- Organização: Os dados coletados das entrevistas em papel foram eletronicamente escritos e organizados logo após as entrevistas foram conduzidas. Os dados de todas as entrevistas foram alinhados para melhorar a análise;

- Codificação: foi utilizada em cada grupo para permitir uma descrição sistemática e precisa dos resultados oriundos do estudo de campo. Assim, dados referentes aos elementos conceituais envolvidos na aplicação da modularidade e das trajetórias da relação MID e MIP foram reduzidos e codificados. Esse procedimento foi importante para estabelecer padrões para triangulação de dados adicionais;

- Identificação de inter-relações: este passo ajudou a identificar as relações entre os elementos conceituais encontrados durante o estudo. Construiu-se um mapa para evidenciar os padrões e as relações entre os conceitos, buscando organizar os dados para se estabelecer inter-relações entre os elementos conceituais da literatura e as evidências da pesquisa de campo;

- Inferência: por meio das inferências a partir dos dados coletados e ordenados, identificou-se os elementos conceituais que emergiram do campo. Posteriormente, estabeleceu-se como MID e MIP se conectam, comparando-se os resultados com a literatura pertinente no tema, viabilizando a análise da relação entre MID e MIP na empresa investigada.

A próxima seção apresenta os resultados deste estudo. 


\section{RESULTADOS}

Esta seção é subdividida em: (i) objetivos da modularidade, benefícios e desvantagens, e; (ii) relação MID-MIP na empresa investigada.

\subsection{Contexto relativo aos objetivos, benefícios e desvantagens da modularidade}

A empresa passou a aplicar a modularidade em seus produtos e processos, de forma mais intensa em 2006-2007, focada na redução de custos e tempo de mercado. Quanto a divisão de módulos, os módulos principais fabricados pela empresa foram: assentos, para-choques, frontend, cockpit, portas e tanque de combustível. Tais módulos foram utilizados em uma plataforma de produto composta por quatro modelos de veículos diferentes em termos de engenharia, arquitetura de veículo e faixas de mercado.

O 'número de módulos' e 'quais módulos' para construir cada veículo são os mesmos para cada veículo, e dentro de cada um, existem requisitos específicos a serem cumpridos. Assim, tanto o fabricante de automóveis como os fornecedores definem as especificações do produto. Entretanto, a montadora decide todas as estratégias para modularizar seus produtos e define o cronograma para a produção e entrega do módulo. Os fornecedores desenvolvem a tecnologia e outras características necessárias para cumprir com as demandas da montadora.

Como principais benefícios da aplicação de modularidade, a montadora destacou que essa facilitou a manutenção do produto, reduziu o número de fornecedores que trabalham com a empresa e o número de itens em estoque. Adicionalmente, a montadora identificou outros benefícios, como ajudar no desenvolvimento de novos produtos através do projeto de novos módulos, reduzindo o tempo de entrega, facilitando as atualizações de produtos através da mudança de módulos, aumentando o envolvimento dos fornecedores no desenvolvimento de novos produtos e mais personalização do produto.

No entanto, a montadora também apontou algumas desvantagens na aplicação a modularidade. O baixo desempenho geral dos veículos devido ao uso de módulos "genéricos" foi a primeira questão destacada. Outros desafios da implementação da modularidade incluem: risco de perder o controle de algumas atividades de projeto, inibir o projeto do módulo para as capacidades dos fornecedores atuais, encontrar fornecedores especializados para fabricar os módulos, mudar de uma cadeia de suprimentos tradicional para uma modular e transferir atividades menos importantes para os fornecedores.

\subsection{Modularidade no design e modularidade nas relações de produção}

O fabricante de automóveis aplica a MID e (com menos intensidade) a MIP. Isso foi evidenciado a partir da estruturação dos projetos da montadora: a partir da definição das funções e sub-funções dos veículos, define-se quais módulos vão desempenhar essas funcionalidades e também quais serão os fornecedores responsáveis pelos mesmos. A partir dessas definições no projeto e dos sistemas e módulos que compõem os veículos da plataforma, a montadora organiza e estrutura a produção, conforme as necessidades de projeto. Nesse sentido, MIP se torna uma consequência de MID, caracterizando-se assim a existência de relações entre MID e MIP, com trajetória predominante de MID para MIP.

Em relação a conexão MID-MIP, a montadora aponta que a importância dessa é evidenciada no conceito da empresa, denominado "sistema produto-processo". A principal motivação para a trajetória predominante de MID para MIP é que modificações de projeto têm custos maiores envolvidos comparando com modificações na produção. Assim, elementos como a comunalidade e funcionalidade são influentes na conexão entre MID e MIP. Portanto, pode-se 
sugerir que, nesta planta, a MIP é condicionada pelo que é feito durante a modularização no projeto.

A montadora aplica MID permitindo MIP por meio de uma redução no tempo de montagem do veículo, uma vez que a arquitetura do produto modular é definida considerando as capacidades de produção e os investimentos necessários para a viabilidade dos veículos projetados. Houve também maior flexibilidade na produção, pois é necessário tornar os processos de fabricação e as linhas de montagem mais facilmente ajustáveis para produzir as variantes demandadas dos módulos e veículos da plataforma desenhada.

Quanto ao relacionamento entre MID e MIP na perspectiva das montadoras e fornecedores, os fornecedores seguem como especialistas em termos de construção de módulos e atendimento aos requisitos específicos exigidos pela montadora, enquanto a montadora avalia como os módulos se adequam ao produto como um todo, e também se os módulos atendem aos requisitos estabelecidos do produto.

\section{DISCUSSÃO}

Acerca dos objetivos de modularidade, a maior parte das evidências sugere que a empresa investigada se concentra em reduzir custos e lead-time. A empresa pretende reduzir os custos principalmente por meio do uso de componentes similares nas variantes da plataforma de produto, bem como implementar mais automatização nas linhas de montagem, estabelecendo um número fixo e mais genérico de módulos para todos os veículos. Entretanto, nota-se que esses módulos genéricos trazem dificuldades quanto ao desempenho de alguns dos veículos projetados. O módulo do motor, por exemplo, é o mesmo para as quatro variantes de veículos da plataforma, sendo que um deles, de maior porte e peso, tem desempenho técnico limitado devido a essas circunstâncias.

A montadora investigada, de modo geral, ainda decide as estratégias de modularização dos seus veículos e definem isso, principalmente, a partir da plataforma de produto. $\mathrm{O}$ estabelecimento das estratégias considera as funções do produto, distribuídas pelos módulos, que também têm suas interfaces definidas pela montadora. As evidências empíricas indicam que a montadora investigada foca em analisar como módulos desenvolvidos pelos fornecedores irão cumprir com todas as variantes desenvolvidas pela montadora. Nesse sentido, o argumento de perda de aprendizagem no desenvolvimento, porque os engenheiros fornecedores tornam-se mais especializados (ZIRPOLI; BECKER, 2011), é suportado. No entanto, a empresa não está mais centrando esforços em construir os módulos. A montadora analisa se os fornecedores cumprem os requisitos para cada arquitetura de veículo, enquanto os fornecedores trabalham em como realizar as demandas da montadora examinada.

Destaca-se também a importância da relação entre MID e MIP, bem como sua trajetória (de MID para MIP). Nesse sentido, embora a montadora argumente que estabelecem relações entre MID e MIP, foi possível observar que é dada mais atenção às práticas de MID (corroborado por JACOBS et al., 2011; PARALIKAS et al., 2011; LUCARELLI et al., 2015). Enquanto a MID é explorada em termos de comunalidade, padronização, terceirização, plataforma de produtos e decisões de engenharia com fornecedores, as mudanças com relação a MIP se concentram em automatizar processos e configurar os processos de produção, separando-os em módulos de trabalhos manuais e automatizados. Assim, houve benefícios em termos de especificações de projeto e de desenvolvimento do mesmo, no entanto as alterações na produção foram limitadas, uma vez que a estrutura produtiva já existia e necessitou de mudanças incrementais em termos de tecnologias e adequações de layout, para que os processos se tornassem mais autônomos e independentes. 
Assim, pode-se sugerir que, embora haja mudanças na MIP por causa da MID, essas mudanças ainda são limitadas devido ao maior foco na MID do que na MIP. Outro motivo para essas mudanças limitadas é o fato de a estrutura de produção da montadora analisada não se tratar de um novo arranjo produtivo. Com isso, as alterações em termos de MIP são mais incrementais do que radicais.

\section{CONCLUSÕES}

Este trabalho analisou a relação existente entre a MID e a MIP, buscando identificar os principais constructos norteadores dessa potencial conexão. A empresa investigada aplica conceitos que acabam por estabelecer uma relação entre MID e MIP, com uma trajetória predominante da MID para a MIP, por meio de elementos conceituais mais influentes nas decisões de projeto de produto, que posteriormente são direcionadoras das alterações na produção, sob a lógica modular. No entanto, conclui-se as mudanças relacionadas a MIP foram limitadas. Assim, sugere-se que é importante estabelecer, previamente, objetivos claros quanto a adoção da modularidade e, a partir disso, o alinhamento estratégico e operacional entre a MID e a MIP pode ser importante para se obter mais vantagens a partir da estratégia modular.

Quanto às limitações deste estudo, destaca-se que um estudo de caso único foi conduzido, portanto a generalização dos resultados é limitada. Outros estudos de campo em diferentes montadoras precisam ser realizados para aumentar a validação externa desses resultados. Assim, surge como oportunidade futura de pesquisa expandir a pesquisa analisando outras montadoras. Além disso, a condução de casos múltiplos possibilita a identificação de fatores contingenciais influentes na adoção da modularidade nas empresas envolvidas na cadeia automotiva, sejam essas montadoras ou fornecedores.

\section{REFERENCIAS}

BARDIN, L. Content analysis. Lisboa: Editions. 1977.

CAMPAGNOLO, D.; CAMUFFO, A. What really drives the adoption of modular organizational forms? An institutional perspective from Italian industry-level data. Industry and Innovation, v. 16, n. 3, p. 291-314, 2009.

CAMPAGNOLO, D.; CAMUFFO, A. The concept of modularity in management studies: A literature review. International Journal of Management Reviews, v. 12, n. 3, p. 259-283, 2010.

FIXSON, S.K. Modularity and commonality research: past developments and future opportunities. Concurrent Engineering Research and Applications, v. 15, n. 2, p. 85-111, 2007.

FIXSON, S.K.; PARK, J.K. The power of integrality: Linkages between product architecture, innovation, and industry structure. Research Policy, v. 37, n. 8, p. 1296-1316, 2008.

HOETKER, G. Do modular products lead to modular organizations? Strategic Management Journal, v. 27, n. 6, p. 501-518, 2006. 
JACOBS, M., DROGE, C.; VICKERY, S.K.; CALANTONE, R. Product and Process Modularity's Effects on Manufacturing Agility and Firm Growth Performance. Journal of Product Innovation Management, v. 1, n. 1, p. 123-137, 2011.

LUCARELLI, M.; MATT, D.T.; RUSSO SPENA, P. Modular architectures for future alternative vehicles. International Journal of Vehicle Design, v. 67, n. 4, p. 368-387, 2015.

MIKKOLA, J.H. Capturing the Degree of Modularity Embedded in Product Architectures. Journal of Product Innovation Management v. 23, n. 2, p. 128-146, 2006.

MILES, M.B.; HUBERMAN, A.M. Qualitative Data Analysis. London: Sage Publications, London. 2014.

PARALIKAS, J.; FYSIKOPOULOS, A.; PANDREMENOS, J.; CHRYSSOLOURIS, G. Product modularity and assembly systems: An automotive case study. CIRP Annals Manufacturing Technology, v. 60, n. 1, p. 165-168, 2011.

PASCHE, M.; SKÖLD, M. Potential drawbacks of component commonality in product platform development. International Journal of Automotive Technology and Management, v. 12, n. 1, p. 92-108, 2012.

RO, Y.K.; LIKER, J.K.; FIXSON, S.K. Modularity as a Strategy for Supply Chain Coordination: The Case of U.S. Auto. IEEE Transactions on Engineering Management, v. 54, n. 1, p. 172-189, 2007.

SANCHEZ, R. Building real modularity competence in automotive design, development, production, and after-service. International Journal of Automotive Technology and Management, v. 13, n. 3, p. 204-236, 2013.

SHAMSUZZOHA, A.H.M., HELO, P.T.; KEKALE, T. Application of modularity in world automotive industries: a literature analysis. International Journal of Automotive Technology and Management, v. 10, n. 4, p. 361-377, 2010.

STONE, R.B.; WOOD, K.L.; CRAWFORD, R.H. A heuristic method for identifying modules for product architectures. Design Studies, v. 21, n. 1, p. 05-31, 2000.

TAKEISHI, A.; FUJIMOTO, T. Modularization in the car industry: Interlinked Multiple Hierarchies of Product, Production and Supplier Systems. In: PRENCIPE, A.; DAVIES, A.; HOBDAY, M. (eds). The Business of Systems Integration. Oxford Scholarship Online, p. 01-24, 2003.

VOSS, C. Case Research in Operations Management. In: KARLSSON, C. (ed.). Researching Operations Management. Londres: Taylor \& Francis. 2009.

YIN, R.K. Case Study Research: Design and Methods. $5^{\text {a }}$ Ed. California: Sage Publications. 2014.

ZIRPOLI, F.; BECKER, M.C. The limits of design and engineering outsourcing: Performance integration and the unfulfilled promises of modularity. R\&D Management v. 41, n. 1, p. 2143, 2011. 